\section{Advancing leukemia diagnos- tics: Role of Next Generation Sequencing (NGS) in acute myeloid leukemia}

\author{
Torsten Haferlach \\ MLL Munich Leukemia Laboratory, \\ Munich, Germany
}

\begin{abstract}
AML diagnostics, initially based solely on morphological evaluation, now relies on multiple disciplines to reach its full potential. Only by integrating the results of cytomorphology, cytochemistry, immunophenotyping, cytogenetics and molecular genetics it is possible to fulfil WHO classification and ELN prognostication systems. Especially molecular genetics has gained a lot of interest over the last decade, mainly through the introduction of next generation sequencing (NGS). NGS application ranges from the investigation of single genes and panels to even whole exomes, transcriptomes and genomes. In routine AML diagnostics panels are the preferred NGS methodology. Here, we will review the power and limitations of NGS in the context of diagnosis, prognosis and precision medicine. Due to high dimensionality, NGS data interpretation is challenging but it also offers a unique investigatory chance and the opportunity to apply data mining techniques such as artificial intelligence. We will also reflect on how the incorporation of the improved knowledge base into routine diagnostics can pave the way for better treatment and more cure in AML.
\end{abstract}

\section{Introduction}

Different methods are available to diagnose and to follow up in patients with AML. The molecular methods have been dramatically improved over the last 20 years, paralleled by a significant knowledge growth. As a result, a lot of fascinating research has been conducted and the resulting insights and optimized technologies were gradually introduced into routine diagnosis and prognosis of AML. In order to always provide the best possible diagnosis and treatment for the patients constant updates are of prime importance. We here demonstrate state-of-the-art of NGS for routine diagnostics in AML at diagnosis, during follow up and for detection of measurable residual disease (MRD). We also will cover some aspects of data handling and the respective turnaround times to be fulfilled in an acute leukemia setting. Let's first start to discuss the background for NGS applications in AML.

\section{Current routine diagnostic workup in AML}

\section{Cytomorphology}

Cytomorphology is the first step in the diagnostic evaluation of hematological diseases. Due to its fast turnaround time, targeted downstream analysis can be initiated, allowing for a time- and cost-effective stepwise diagnostics. In AML, cytomorphology is indispensable to diagnose the disease and differentiate it from other hematological neoplasms, to identify the subtype according to WHO classification and to monitor disease kinetics and response. A comprehensive and complementary insight into the tissue context can be gained by bone marrow biopsy, especially with respect to the cellularity, the histotopography, and the proportion and differentiation state of hematopoietic cells. ${ }^{1}$

\section{Multiparameter flow cytometry (MFC)}

Multiparameter flow cytometry (MFC) is used to detect, characterize and quantify normal and malignant cell populations. In addition to its value for diagnostics and differential diagnostics, the identification of aberrant immunophenotypic features of leukemic cells allows for the sensitive MRD monitoring in AML.

\section{Cytogenetics}

Cytogenetics comprises the methods of chromosome analysis and fluorescence in situ hybridization (FISH). In AML, cytogenetics allows the diagnosis of cytogenetically defined subtypes. Aside to its relevance for WHO classification, the presence of certain cytogenetic abnormalities strongly influences prognosis which is considered e.g. in the ELN risk stratification system. ${ }^{2}$ As for cytomorphology and MFC, cytogenetics can contribute to response assessment and monitoring of disease kinetics. Moreover, cytogenetic analysis provides insight into patterns of clonal evolution.

\section{Molecular genetics}

Molecular genetics have led to an unparalleled gain in our understanding of AML pathobiology. This knowledge has been incorporated into classification, prognostication, MRD monitoring, and has guided the emerging development and
Correspondence: Torsten Haferlach, MLL Munich Leukemia Laboratory, 81377 Munich, Germany.

E-mail: torsten.haferlach@mll.de

Key words: acute myeloid leukemia, multidisciplinary diagnostics, next generation sequencing, precision medicine.

Conflict of interest: the author declares no potential conflict of interest.

This work is licensed under a Creative Commons Attribution-NonCommercial 4.0 International License (CC BY-NC 4.0).

(C) Copyright: the Author(s), 2020

Licensee PAGEPress, Italy

Hematology Reports 2020; 12(s1):8957

doi:10.4081/hr.2020.8957

implementation of precision medicine. With respect to diagnostic techniques, PCR (polymerase chain reaction) and NGS represent the gold standard. By PCR a known target sequence is specifically amplified, allowing for its detection (endpoint PCR) as well its quantification (quantitative PCR, qPCR). By employing a preceding reverse transcription step, transcripts can be equally detected and quantified by PCR-based approaches. Given their high sensitivity of up to $10^{-6}$ PCR-based assays are of especially great value in MRD diagnostics. For sequence determination, next generation sequencing has replaced older methods such as Sanger sequencing in the clinical routine. Compared to Sanger sequencing, NGS provides a higher sensitivity, as well as the capacity for massive parallelization. Within just one sequencing run, a large number of samples and/or genomic loci can be examined.

\section{Applications of NGS}

The capacity for massive parallel sequencing is commonly used for targeted NGS panel testing, which currently represents the state-of-the-art NGS application in the clinical routine. Such a panel can for example encompass all genetic loci that are associated with a given disease or are relevant for classification, prognostication and/or therapeutic decision making. One of the advantages of gene panels is the possibility to extent the panel when new findings are made. In addition to recurrently mutated single nucleotide variants ( $\mathrm{SNVs}$ ) and small insertions/deletions (indels), targeted sequencing approaches can also capture translocations if the breakpoints are known. It is equally possible to include a selection 
of single nucleotide polymorphisms (SNPs) that are spaced evenly across the entire genome to assess the copy number state. Such a comprehensive panel would allow a refinement of risk stratification in AML and consequently treatment decisions.

As NGS is a highly versatile platform, non-targeted approaches can also be implemented. Sequencing the whole genome (WGS), the whole exome (WES) and the whole transcriptome (WTS), not only provides an unparalleled insight into the landscape of sequence variations, but also enables detection of numerical and structural abnormalities (WES/WGS) as well as the detection of fusion transcripts, and analysis of gene expression (WTS). AML diagnostics will especially benefit from streamlining genetic characterization, given the role of mutations and gene fusions in pathogenesis and their relevance for classification, prognosis and therapy. With reduction of sequencing costs and increase in computing power, we will observe the transition of such genome wide NGS applications from research to routine diagnostics in next 5 years.

\section{The NGS workflow and its tech- nical aspects}

The NGS wet-lab workflow contains multiple time-sensitive steps for quality assurance and several factors might prevent the success of the approach. Hence, to meet the necessary quality requirements a high level of standardization is desirable to reduce manual bias and to increase homogeneity, reproducibility and efficacy. The increased reproducibility is what makes the comparison of different processing batches possible.

In most specialized hematologic laboratories peripheral blood or bone marrow aspirates are the gold standard materials to perform NGS. The purity and quality of the extracted nucleic acid impacts the reliability of NGS results and is influenced by the sample transport time and condition. DNA is a rather stable molecule and can easily be stored at room temperature. However, due to the lysis of white blood cells the tumor to healthy cell ratio might be distorted, potentially limiting the sensitivity over time. RNA is less stable and fast sample processing is crucial to ensure an unbiased assessment of a patient's transcriptome.

Various library kits for different platforms are available, ranging from all-in-one kits that cover everything from DNA extraction to purification to specialized library preparation kits that use fragmented
DNA to prepare purified libraries. DNA fragmentation can be performed either enzymatically or mechanically. Enzymatic fragmentation loses less DNA material during the process compared to mechanic fragmentation but it is also more strongly affected by sequence context such as GC-rich regions. Although some DNA regions might break more easily than others, the mechanic fragmentation results in libraries with higher complexity and more uniformly covered genomic regions. For WTS libraries either polyA + enrichment or rRNA depletion techniques are used to remove the highly abundant - but not very informative - ribosomal RNAs, followed by cDNA synthesis and PCR amplification. rRNA depletion methods capture the complete transcriptome, including novel and known non-coding RNAs, whereas polyA+ selected samples show a higher exonic coverage due to an increased fraction of reads mapping to the coding region.

The economically favorable multiplexing strategy of NGS requires the addition of unique index sequences per sample during library preparation. Incorrect assignment of libraries from the expected index to a divergent index (= index hopping) has been a known phenomenon. Its impact can be reduced by using unique dual indexing pooling combinations to eliminate hopped reads from downstream analysis. For PCRbased libraries unique molecular identifiers (UMI) can be added to identify PCR artifacts, enabling a more accurate detection of true variants.

Reliable detection of somatic variants is mainly influenced by the coverage and tumor cell content. Currently, no standardized recommendations of a minimum coverage for a given detection limit exist. It has to be taken into account that technical bias in targeted sequencing assays leads to a skewed coverage distribution with coverage drops in GC-rich regions, demanding higher sequencing capacities. The sensitivity is further limited by the sequencing error rate which depends on the used platform, the GC content and the read length and ranges mostly between 0.1 and $1 \%$. In general, with 10 variant supporting reads and a coverage of $100 \mathrm{x}$, the detection limit is set to $\sim 10 \%$ variant allele frequency (VAF) and, hence, panel-based approaches usually target a coverage $>1,000 x$.

A definite differentiation between rare pathogenic germline alterations and leukemia-related somatic mutations is only possible by sequencing matched germline/tumor samples. However, in practice the availability of control samples derived from non-hematopoietic and nonmalignant tissue is often challenging. Also because there is a high risk for contamination with blood cells in many conventional control tissues. For frequently mutated genes in AML both somatic mutations and pathogenic germline mutations are known (e.g. $R U N X 1$ ) and, thus, there is a considerable risk for misinterpretation.

\section{NGS: Making sense of the data}

Introduction of NGS and an increasing awareness for the genetic complexity of AML have heralded a shift from the investigation of single genes to the evaluation of a comprehensive panel of genomic loci. The increase in sequencing sample size naturally also correlates with an increase in the time and effort required for evaluation of NGS data. Variant calling represents a major challenge in today's routine diagnostics, and requires great expertise and often time-consuming comparison of detected variants with information available in different databases (e.g. ClinVar, COSMIC, gnomAD). Some AML-associated genes are known to show mutational hotspots (e.g. FLT3, IDH1, IDH2), while others exhibit a diverse mutational profile (e.g. TET2). For many gene variants the distinction between 'pathogenic' and 'non-pathogenic' variants thus poses a challenge, and in some cases only the categorization as 'variant of unknown significance' remains, either due to missing data/lack of characterization or due to contradictory data. Artificial intelligence (AI) has been shown to streamline this process by integration of publicly available information and prediction of variant pathogenicity. ${ }^{3}$ With the advance of genome wide NGS approaches, storing, processing and interpreting the data will be an emerging and immense challenge, which requires assistance through an armamentarium of hardware and software solutions as well as sophisticated bioinformatic tools and workflows. Data mining techniques such as AI will be necessary to identify clinically meaningful genetic patterns of disease and to map genetic interactions.

\section{Implementation of NGS in state- of-the-art AML diagnostics}

\section{NGS in mapping the molecular landscape of AML}

In AML, cytogenetic abnormalities have long been recognized for definition of disease, their prognostic impact and/or clinical relevance. However, only between 50 
and $60 \%$ of patients carry cytogenetic abnormalities. ${ }^{4}$ In contrast to this, comprehensive panel testing for mutations in several large scale studies allowed the identification of at least one mutation in $\geq 90 \%$ of AML patients. ${ }^{5-8}$ The majority of mutations falls within distinct functional classes and leads to perturbation of tumor suppression, DNA methylation, chromatin modification, cell signalling, cohesion, splicing and/or transcriptional regulation. Nucleophosmin 1 (NPM1), which defines an AML subtype, represents an additional mutation class. ${ }^{7}$ In most AML patients $(86 \%$ in the study by Papaemmanuil et al. 2016) more than one mutation is detectable, ${ }^{8}$ with up to 13 different mutations per AML patient. ${ }^{7}$

This knowledge of the genetic complexity as well as the analysis of mutational load indicated certain leukemogenic trajectories and suggested that AML pathogenesis is a multi-step process. . $^{1,9}$ Without the massive parallelizability of NGS, the mapping of the molecular landscape would not have been possible. However, given the targeted nature of panel-based testing and its strong focus on mutational analysis, there is likely much to be learned from non-targeted genome wide approaches that provide information on sequence as well as on structure, copy number and gene expression.

\section{NGS at diagnosis}

For classification of a case according to the WHO classification (2017) the mutational status of three genes must be determined: NPM1, RUNX1 and CEBPA. ${ }^{1}$ NPM1 and $C E B P A$ both have been associated with a favorable prognosis, however the genetic context has to be taken into consideration. In case of AML with NPM1 mutation, a concomitant internal tandem duplication within the FLT3 gene (FLT3-ITD) abolishes the favorable prognostic impact of a NPMI mutation. ${ }^{1}$ In case of $C E B P A$, both alleles have to be mutated to confer the favorable prognostic effect, hence the WHO category 'AML with biallelic mutation of CEBPA'. ${ }^{1}$ NPM1 mutations and biallelic CEBPA mutations retain their prognostic power in the presence of multilineage dysplasia, this is but just one example why a careful and comprehensive genetic analysis is imperative, even in the presence of well-defined morphological characteristics. ${ }^{1}$ While AML with mutated NPM1 and AML with biallelic mutations of CEBPA are established subtypes within the 2017 WHO classification, AML with mutated $R U N X 1$ is a provisional entity. ${ }^{1}$

AML diagnostics at diagnosis should not be limited to mere classification of a case, given that risk stratification is of great clinical importance in AML and that genetic lesions exert the strongest influence on prognosis. Risk stratification according to the ELN recommendations requires knowledge on the mutational status of five genes: NPM1, CEBPA, FLT3, ASXL1, TP53 and $R U N X 1 .^{2}$ As described above the presence of a FLT3-ITD mutation modulates the prognosis of AML patients with a NPM1 mutation. Moreover, mutational load appears to be a critical factor, which is why the ELN recommends a cut-off of 0.5 for the allelic ratio of mutant to wildtype FLT3. The prognostic effect of mutations within the tyrosine kinase domain of FLT3 (FLT3TKD) appears to be less conclusive ${ }^{10}$ and FLT3-TKD mutations are thus not considered in the ELN risk stratification. ${ }^{2} R U N X 1$, TP53 and ASXL1 mutations all confer an unfavorable prognosis as do FLT3-ITD mutations (at a high allelic ratio of $>0.5$ in the absence of NPM1 mutations). ${ }^{2}$ Relapse risk in patients that carry these unfavorable molecular markers is high, identifying potential candidates for allogeneic stem cell transplantation. ${ }^{11,12}$

Recent years have brought about a shift in AML therapy, from the standard chemotherapy regimen towards individualized therapeutic strategies. Currently, these are tailored to specific features, i.e. CD33 positivity (gemtuzumab ozogamicin, an anti-CD33 immune conjugate), to specific AML subtypes, i.e. t-AML and AML-MRC (CPX-351, a liposomal formulation of the chemotherapeutics cytarabine and daunorubicin), or to specific genetic lesions, i.e. FLT3 mutations (midostaurin, gilteritinib or sorafenib). Additional small molecular inhibitors are currently in development or undergoing the European marketing author- ization procedure (e.g. IDH inhibitors). While some molecular abnormalities are directly targetable, others impact response to therapy. This also should be considered within the treatment algorithm. For example, patients with KIT mutations in the genetic background of AML with $t(8 ; 21)$ and $\operatorname{inv}(16)$ or $t(16 ; 16)$ had a poorer outcome under treatment with high dose cytarabine than without, specific treatment using dasatinib is possible. ${ }^{13}$

In conclusion, a comprehensive stateof-the art diagnosis in AML needs to provide information on the mutational status of several genes in order to aid classification, prognostication and therapeutic decisions (see also Table 1). NGS panel testing provides the means for fast and parallel interrogation of the respective mutational status in the routine setting. As discussed above, the NGS panel could also be expanded to allow detection of translocations that are relevant to diagnosis and/or prognosis (e.g. RUNX1RUNX1T1, CBFB-MYH11, translocations of KMT2A, DEK-NUP214).

\section{NGS in follow-up and MRD diag- nostics}

Therapy in AML has two consecutive aims, 1) achievement of a complete remission (CR) by induction therapy and 2) erasure of residual disease and prevention of relapse through consolidation therapy. While CR is defined solely by clinical and morphological parameters, $\mathrm{CR}_{\mathrm{MRD}}$ (complete remission without minimal/measurable residual disease) was introduced to reflect the prognostic relevance of residual disease detection in $\mathrm{AML},{ }^{2}$ which is linked to a higher relapse risk and reduced survival. ${ }^{14-16}$ While risk stratification according to ELN recommendations at diagnosis, ${ }^{2}$ which is based on detection of cytogenetic and molecular genetic abnormalities, aids choice of induction therapy, both risk evaluation at diagnosis and during follow up are needed for an informed risk adapted postremission therapy.

The prerequisite for MRD diagnostics is a high sensitivity of $10^{-4}$ to $10^{-6}$, which is currently only met by MFC and PCR-based approaches. ${ }^{14}$ In the routine setting, NGS

Table 1. Obligatory mutational analysis at diagnosis.

\begin{tabular}{lll} 
Classification according to WHO1 & Prognostication according to ELN risk stratification ${ }^{2}$ & Therapy \\
- NPM1 & $\bullet$ NPM1 & $\bullet$ FT3-ITD \\
- Biallelic CEBPA & $\bullet$ Biallelic CEBPA & $\bullet$ KIT \\
- RUNX1 & $\bullet$ RUNX1 & \\
& $\bullet$ ASXL1 & \\
& $\bullet$ FLT3-ITD \\
& $\bullet$ TP53 & \\
\hline
\end{tabular}


can be used to identify potential MRD markers, and PCR-based approaches are subsequently used for their quantification. However, despite the relatively low sensitivity of the method (of $\sim 1 \%$ mutational load), ${ }^{14}$ several studies have demonstrated the clinical value of NGS-based MRD detection in the post-induction ${ }^{5,6,17}$ and the pre-transplant ${ }^{16,18-21}$ setting. ${ }^{22}$ With efforts to optimize both experimental parameters and the bioinformatic workflow, ${ }^{16,19,21,22}$ MRD monitoring might be among future NGS applications in AML diagnostics.

Today, well-established and recommended markers for molecular MRD assessment are: RUNX1-RUNX1T1, CBFBMYH11, PML-RARA and NPM1, ${ }^{14}$ which limits molecular MRD diagnostics to a subset of AML cases. In general, potential molecular MRD markers fall within three categories: ${ }^{14}$

- Fusion gene transcripts

- Somatic mutations

- Aberrant gene expression

In theory, especially with the possibilities of genome wide approaches, a comprehensive NGS analysis would allow the identification of a potential MRD marker in almost every case.

However, in clinical routine, there are several caveats to molecular genetic MRD monitoring.

Not every marker is suitable for MRD monitoring, whether for technical or biological reasons.

For some markers the necessary sensitivity cannot be achieved, this is for example the case with quantification of WT1 expression levels ${ }^{14}$. As an example for confounding biology, there are genetic loci susceptible to chromosomal losses or gains at relapse (e.g. FLT3 mutations). ${ }^{14}$ Genes for which germline mutations as well as somatic mutations have been described can cause misinterpretation in residual disease monitoring (e.g. RUNX1). ${ }^{14}$ While often found in patients with AML, DNMT3A, ASXL1 and TET2 mutations are associated with clonal hematopoiesis of indeterminate potential, an age-related condition. ${ }^{23-26}$ They do not represent suitable MRD markers, since mutations in all three genes have been described to persist, even when a complete remission is achieved ${ }^{5,27}$.

Due to these limitations, the use of most molecular mutations as sole MRD marker is not recommended. ${ }^{14}$ However, due to the genetic complexity of the disease, NGS allows the identification of more than one mutation per patient in the majority of cases, and their parallel monitoring might represent a suitable strategy. ${ }^{22}$

In case of a relapse, a comprehensive genetic characterization including muta- tional analysis has to be repeated, because the genetic and clonal landscape may differ between initial diagnosis and relapse.

\section{NGS in precision medicine}

The earliest example of precision therapy in AML is the treatment algorithm for acute promyelocytic leukemia (APL), caused by the $P M L-R A R A$ fusion. Due to a high hemorrhagic risk, this AML subtype has to be diagnosed within hours to initiate immediate treatment. The vast majority of patients achieves long lasting remission under treatment with all-trans retinoic acid (ATRA) and arsenic trioxide. ${ }^{28-30}$ Monitoring of $P M L-R A R A$ transcript levels is of clinical value, as pre-emptive therapy in MRD positive patients reduces relapse risk. ${ }^{31}$

NGS is not equipped to deliver findings within a matter of hours, and thus not relevant for the diagnosis of APL. However, with the increasing consideration of genetic background and the emerging necessity to identify targetable genetic lesions, NGS, with a turnaround time of $<7$ days, is the ideal method for ensuring the balance between a most comprehensive analysis and a fast processing time for diagnostics in other AML subtypes.

The FLT3 inhibitor midostaurin is the first example of an approved drug that targets a molecular mutation. Addition of midostaurin to the standard chemotherapy regimen has been shown to improve outcome for FLT3 mutated AML patients. ${ }^{32}$ For planning the suitable post-remission treatment algorithm in FLT3 mutated AML, several parameters should be considered: the MRD status of a potential concomitant NPM1 mutation, ${ }^{33,34}$ the type of FLT3 mutation (ITD or TKD) $)^{10,35,36}$ as well as the allelic ratio of a FLT3-ITD mutation. ${ }^{37}$ Moreover, maintenance therapy with the FLT3 inhibitor sorafenib was shown to improve outcome in the post-allogeneic stem cell transplantation setting for FLT3ITD mutated AML. ${ }^{38}$

In the past, NGS-based mapping of the molecular genetic landscape has led to identification of several lesions targetable by small molecule inhibitors (summarized in Döhner et al. 2017) $)^{2}$, which are currently being developed, evaluated in clinical trials, or as is the case for IDH inhibitors under review for marketing authorization. In the future, it is highly likely that non-targeted genome wide studies will discover novel targetable abnormalities and aberrant pathways.

The term 'precision medicine' covers not only the directed therapeutic agents but also the accompanying diagnostics. The latter includes identification of targetable genetic lesions and characterization of the genetic background for diagnosis, risk stratification and therapy planning, response monitoring during follow-up as well as identification of resistance mechanisms and new potential targets in case of a relapse. Through the introduction of targeted therapies, NGS will continue to gain importance in the clinical routine.

\section{Conclusions}

NGS driven characterization of the molecular genetic landscape in AML has advanced not only our understanding of the disease but also classification, prognostication and therapy. Today, the mutational status of several AML-associated genes has to be considered at diagnosis, making NGS panel testing the method of choice in the routine setting. The potential of the technique, however, is far from exhausted, and several studies have demonstrated the clinical value of NGS-based MRD evaluation. In addition to this, the need for comprehensive molecular diagnostics will go hand in hand with further development and implementation of targeted therapies in AML treatment algorithms. Finally, genome wide NGS approaches, such as WES, WGS and WTS will help us to go beyond of what is known today about clinically relevant genetic changes, with further implications for classification, prognosis and therapy.

\section{References}

1. Swerdlow SH, Campo E, Harris NL, et al. WHO classification of tumours of haematopoietic and lymphoid tissues. Revised 4th edition ed. International Agency for Research on Cancer Lyon 2017.

2. Döhner H, Estey E, Grimwade D, et al. Diagnosis and management of AML in adults: 2017 ELN recommendations from an international expert panel. Blood. 2017;129(4):424-447.

3. Hutter S, Baer C, Walter W, Kern W, Haferlach C, Haferlach T. A Novel Machine Learning Based in silico Pathogenicity Predictor for Missense Variants in a Hematological Setting. Blood. 2019;134(Supplement_1):20902090.

4. Kumar CC. Genetic abnormalities and challenges in the treatment of acute myeloid leukemia. Genes Cancer. 2011;2(2):95-107.

5. Jongen-Lavrencic M, Grob T, Hanekamp $\mathrm{D}$, et al. Molecular Minimal Residual 
Disease in Acute Myeloid Leukemia. New England Journal of Medicine. 2018;378(13):1189-1199.

6. Morita K, Kantarjian HM, Wang F, et al. Clearance of Somatic Mutations at Remission and the Risk of Relapse in Acute Myeloid Leukemia. Journal of Clinical Oncology. 2018;36(18):17881797.

7. Network TCGAR. Genomic and Epigenomic Landscapes of Adult De Novo Acute Myeloid Leukemia. New England Journal of Medicine. 2013;368(22):2059-2074.

8. Papaemmanuil E, Gerstung M, Bullinger L, et al. Genomic Classification and Prognosis in Acute Myeloid Leukemia. N Engl J Med. 2016;374(23):2209-2221.

9. Thiede C. Impact of mutational analysis in acute myeloid leukemia. Hematology Education: the education programme for the annual congress of the European Hematology Association. 2012;6:8.

10. Bacher U, Haferlach C, Kern W, Haferlach T, Schnittger S. Prognostic relevance of FLT3-TKD mutations in AML: the combination matters - an analysis of 3082 patients. Blood. 2008;111(5):25272537.

11. Appelbaum FR, Gundacker H, Head DR, et al. Age and acute myeloid leukemia. Blood. 2006;107(9):3481-3485.

12. Cornelissen JJ, Gratwohl A, Schlenk RF, et al. The European LeukemiaNet AML Working Party consensus statement on allogeneic HSCT for patients with AML in remission: an integrated-risk adapted approach. Nat Rev Clin Oncol. 2012;9(10):579-590.

13. Tallman MS, Wang ES, Altman JK, et al. Acute Myeloid Leukemia, Version 3.2019, NCCN Clinical Practice Guidelines in Oncology. 2019;17(6):721.

14. Schuurhuis GJ, Heuser M, Freeman S, et al. Minimal/measurable residual disease in AML: a consensus document from the European LeukemiaNet MRD Working Party. Blood. 2018;131(12):1275-1291.

15. Grimwade D, Freeman SD. Defining minimal residual disease in acute myeloid leukemia: which platforms are ready for "prime time"? Blood. 2014;124(23):33453355.

16. Hourigan CS, Gale RP, Gormley NJ, Ossenkoppele GJ, Walter RB. Measurable residual disease testing in acute myeloid leukaemia. Leukemia. 2017;31(7):14821490.

17. Klco JM, Miller CA, Griffith M, et al. Association Between Mutation Clearance After Induction Therapy and Outcomes in Acute Myeloid Leukemia. JAMA. 2015;314(8):811-822.

18. Balagopal V, Hantel A, Kadri S, et al.
Measurable residual disease monitoring for patients with acute myeloid leukemia following hematopoietic cell transplantation using error corrected hybrid capture next generation sequencing. PLoS One. 2019; 14(10): e224097-e0224097.

19. Kim T, Moon JH, Ahn J-S, et al. Next-generation sequencing-based posttransplant monitoring of acute myeloid leukemia identifies patients at high risk of relapse. Blood. 2018;132(15):1604-1613.

20. Press RD, Eickelberg G, Froman A, et al. Next-generation sequencing-defined minimal residual disease before stem cell transplantation predicts acute myeloid leukemia relapse. American Journal of Hematology. 2019;94(8):902-912.

21. Thol F, Gabdoulline R, Liebich A, et al. Measurable residual disease monitoring by NGS before allogeneic hematopoietic cell transplantation in AML. Blood. 2018;132(16):1703-1713.

22. Yoest JM, Shirai CL, Duncavage EJ. Sequencing-Based Measurable Residual Disease Testing in Acute Myeloid Leukemia. Front Cell Dev Biol. 2020;8:249-249.

23. Genovese G, Kähler AK, Handsaker RE, et al. Clonal Hematopoiesis and BloodCancer Risk Inferred from Blood DNA Sequence. New England Journal of Medicine. 2014;371(26):2477-2487.

24. Jaiswal S, Fontanillas P, Flannick J, et al. Age-Related Clonal Hematopoiesis Associated with Adverse Outcomes. New England Journal of Medicine. 2014;371(26):2488-2498.

25. Steensma DP, Bejar R, Jaiswal S, et al. Clonal hematopoiesis of indeterminate potential and its distinction from myelodysplastic syndromes. Blood. 2015;126(1):9-16.

26. Xie M, Lu C, Wang J, et al. Age-related mutations associated with clonal hematopoietic expansion and malignancies. Nature Medicine. 2014;20(12):14721478.

27. Höllein A, Meggendorfer M, Dicker F, et al. NPM1 mutated AML can relapse with wild-type NPM1: persistent clonal hematopoiesis can drive relapse. Blood Advances. 2018;2(22):3118-3125.

28. Burnett A, Russell N, Hills R, et al. Arsenic trioxide and all-trans retinoic acid treatment for acute promyelocytic leukaemia in all risk groups (AML17): Results of a randomised, controlled, phase 3 trial. The Lancet Oncology. 2015;16.

29. Lo-Coco F, Avvisati G, Vignetti M, et al. Retinoic Acid and Arsenic Trioxide for Acute Promyelocytic Leukemia. New England Journal of Medicine. 2013;369(2):111-121.

30. Platzbecker U, Avvisati G, Cicconi L, et al.
Improved Outcomes With Retinoic Acid and Arsenic Trioxide Compared With Retinoic Acid and Chemotherapy in NonHigh-Risk Acute Promyelocytic Leukemia: Final Results of the Randomized Italian-German APL0406 Trial. Journal of Clinical Oncology. 2017;35(6):605-612.

31. Grimwade D, Jovanovic JV, Hills RK, et al. Prospective Minimal Residual Disease Monitoring to Predict Relapse of Acute Promyelocytic Leukemia and to Direct Pre-Emptive Arsenic Trioxide Therapy. Journal of Clinical Oncology. 2009;27(22):3650-3658.

32. Stone RM, Mandrekar SJ, Sanford BL, et al. Midostaurin plus Chemotherapy for Acute Myeloid Leukemia with a FLT3 Mutation. New England Journal of Medicine. 2017;377(5):454-464.

33. Krönke J, Schlenk RF, Jensen K-O, et al. Monitoring of Minimal Residual Disease in NPM1-Mutated Acute Myeloid Leukemia: A Study From the GermanAustrian Acute Myeloid Leukemia Study Group. Journal of Clinical Oncology. 2011;29(19):2709-2716.

34. Shayegi N, Kramer M, Bornhäuser M, et al. The level of residual disease based on mutant NPM1 is an independent prognostic factor for relapse and survival in AML. Blood. 2013;122(1):83-92.

35. Röllig C, Beelen DW, Braess J, et al. Onkopedia Leitlinien; Akute Myeloische Leukämie (AML). 2019; https://www.onkopedia.com/de/onkope$\mathrm{dia} /$ guidelines/akute-myeloische-leukae$\mathrm{m}$ aml/@@guideline/html/index.html\#litID 0EYSBG. Accessed 2020/09/07.

36. Heuser M, Mina A, Stein EM, Altman JK. How Precision Medicine Is Changing Acute Myeloid Leukemia Therapy. American Society of Clinical Oncology Educational Book. 2019(39):411-420.

37. Burchert A, Bug G, Finke J, et al. Sorafenib As Maintenance Therapy Post Allogeneic Stem Cell Transplantation for FLT3-ITD Positive AML: Results from the Randomized, Double-Blind, PlaceboControlled Multicentre Sormain Trial. Blood. 2018;132(Supplement 1):661-661.

38. Burchert A, Bug G, Fritz LV, et al. Sorafenib Maintenance After Allogeneic Hematopoietic Stem Cell Transplantation for Acute Myeloid Leukemia With FLT3Internal Tandem Duplication Mutation (SORMAIN). Journal of Clinical Oncology. 2020;38(26):2993-3002. 